\title{
Improved Multiple-Scenario Radio Network Dimensioning for WCDMA
}

\author{
Thiaw Seng Ng, Teong Chee Chuah, Yi Fei Tan \\ Faculty of Engineering (FOE), Multimedia University, Cyberjaya, Malaysia \\ Email: thiawseng@gmail.com, \{tcchuah,yftan\}@mmu.edu.my
}

Received September 12, 2011; revised November 10, 2011; accepted November 29, 2011

\begin{abstract}
In wideband code division multiple access (WCDMA) cellular systems, the coverage radius of a cell depends on its current capacity level. As a result, existing WCDMA radio network dimensioning approaches require that coverage and capacity planning be carried out jointly in an iterative manner in order to obtain the minimum site count needed while fulfilling both coverage and capacity requirements. This requires relatively long computational time, particularly when there are many scenarios or what-if cases to be considered. To overcome this problem, we propose an alternative radio network dimensioning approach where coverage planning and capacity planning can be carried out separately to reduce computational time. Besides, a portion of the values calculated in the initial iteration is preserved in a lookup graph, allowing future what-if analysis to be accomplished rapidly. Simulation results show that, unlike the existing approach, the planning and what-if analysis times of the proposed dimensioning approach are independent of the number of scenarios considered. Lastly, we present a few case studies and show that the proposed dimensioning method can give the same prediction accuracy as the existing method.
\end{abstract}

Keywords: WCDMA; Dimensioning

\section{Introduction}

Motivated by successful mobile broadband subscription uptake in some countries, the cellular communication industry around the world is currently aggressively expanding third-generation (3G) systems using wideband code division multiple access (WCDMA). Given the stiff competition in the telecommunication industry, operators seek fast go-to-market solutions for any new types of service to be introduced. Furthermore, operators need to make informed decisions on network expansion to satisfy performance requirements efficiently. This drives the need for efficient radio network planning and rapid estimation of deployment cost. A typical WCDMA radio network planning process involves three stages: initial dimensioning, detailed planning, and network optimization [1]. Initial dimensioning is a rough radio network planning (RNP) process for estimating the number of networking equipment needed to support the required quality of service (QoS) for a given targeted number of subscribers while fulfilling operators' constraints.

In a practical WCDMA network, initial dimensioning involves forecasting of subscribers and traffic growth followed by dimensioning to yield a network deployment plan for the upcoming years. This is carried out through an iterative process between coverage and capacity plan- ning where the number of base stations needed is calculated [1]. Radio network planners usually face a number of challenges as they need to design networks in a short time frame. Many of these challenges are related to the time-consuming design and planning of multi-service radio networks, and some to the particular aspects of the underlying WCDMA radio access method [1]. Initial dimensioning is conventionally accomplished in an iterative process to fulfil the target coverage and capacity objectives [1-4]. This is both complicated and time consuming especially when all characteristics of the radio interface are to be considered. The combinations of scenarios sometimes can easily exceed 2000 iterations in computing the required number of sites and configurations for different cities, operating periods, and clutter types (i.e., urban or rural areas). Thus if the process of determining the number of sites required is too tedious, it will impact timely rollout of network plan to cater both coverage and capacity requirements. The key concern in initial network dimensioning is delivery of network plan in a timely and cost effective manner while ensuring accuracy. Therefore, a fast dimensioning method is desirable to enable operators to quickly analyze different network scenarios, answer WHAT-IF questions and conduct sensitivity analyses.

To date, different methodologies to simplify network 
dimensioning through modeling and optimization by mathematical and heuristic algorithms have been proposed [1-4]. The work in [1] and [2] adopted a mathematical approach where modeling of multi-service environments is described and implications on system performance are addressed. In [3], a method for dimensioning WCDMA networks with variable loads is presented, where iterations take place for each clutter type, area and subscriber input. In [4], characterization of coverage and capacity limited high-speed packet access networks is made. In [5], the authors proposed algorithms for joint uplink/downlink Universal Mobile Telecommunications System radio planning with the objective of minimizing total power consumption in the network. Most studies on optimized network planning focus on locating base stations according to the best trade-off between network infrastructure costs and service coverage. However, these processes are lengthy, requiring large numbers of iterations for a specific network operating scenario and are not efficient for multiple-scenario radio network dimensioning. Furthermore, these methods cannot be easily implemented in a dynamically changing telecommunication environment where new service types are frequently introduced and individual cell analysis is required. Thus, a more practical dimensioning approach is called for and mobile operators start to seek for a more flexible and simple model for network dimensioning. In [6], a novel dimensioning methodology is developed for the Telstra network in Australia; this method derives environmental parameters that can be used to characterize the radio environment for each individual cell in the network. In [7], tools are developed for analyzing individual cell in the design and dimensioning process, as well as pre-operational optimization of the radio network infrastructure.

In general, network dimensioning becomes more complex and time consuming when more service types such as multi-technology, heterogeneous networks and vehicleto-infrastructure (V2I) need to be considered in network planning. To the best of our knowledge, simplification and complexity reduction for multiple-scenario network dimensioning have not been considered. The present work attempts to fill this gap; it proposes an alternative radio network dimensioning approach where separation of coverage planning and capacity planning is made possible to reduce computational time. Besides, a portion of the values calculated in the initial iteration is preserved in a lookup graph, allowing future what-if analysis to be accomplished rapidly.

Extensive simulations have been carried out to verify the proposed dimensioning method. The outcome of the proposed dimensioning is compared and verified with an existing dimensioning method. The contributions of this paper are summarized as follows: 1) A fast dimensioning method which enables operators to quickly dimen- sion and analyze different network scenarios; 2) An effective framework to answer WHAT-IF questions and conduct sensitivity analyses on each individual cell. Conducting simplified network sensitivity analysis using the proposed dimensioning method can provide engineers with better visibility of network behaviors.

This paper is organized as follows. Section 2 reviews the existing radio network dimensioning process. In Section 3, the proposed dimensioning methodology is described. A network characterization methodology using both coverage and capacity criteria is also described. Section 4 presents multi-scenario network dimensioning case studies using the proposed methodology and validation of the proposed model. This is followed by computational time analysis and performance assessment. We conclude the paper in Section 5.

\section{Review of Existing Radio Network Planning Process}

The dimensioning methodologies in [1-3], [8] require coverage and capacity planning processes to be done in an iterative manner as shown in Figure 1. In this approach, the cell range for both uplink (UL) and downlink (DL) is first calculated via coverage planning to obtain the site count needed for coverage, $C_{\text {cov }}$; followed by capacity dimensioning to obtain the site count needed for capacity, $C_{\text {cap }}$. This is followed by an iterative process to adjust the subscribers in each cell; coverage and capacity dimensioning are repeated until the site counts obtained from both dimensioning are matched. That is, iterations are repeated until a balance between radio coverage and network capacity is reached to achieve optimum design where the largest cell range is obtained with the highest capacity. In this approach, optimum design cannot be achieved if the coverage and capacity requirements are not considered jointly.

\subsection{Coverage Planning}

The dimensioning process starts with coverage planning where the link budget is first calculated before capacity is taken into account. In UL link budget calculation, the maximum UL path loss, $L_{\text {pathmax,UL }}$ is given by

$$
L_{\text {pathmax,UL }}=P_{\mathrm{UL}}-S_{\mathrm{UL}}-G_{b}-I_{\mathrm{UL}}
$$

where $P_{\mathrm{UL}}$ is the maximum terminal output power, $S_{\mathrm{UL}}$ is the base station receiver sensitivity, $I_{\mathrm{UL}}$ is the UL interference margin, while $G_{b}$ is the sum of all the gains and losses between the terminal and the base station which comprises the body loss $L_{\mathrm{BL}}$, the building penetration loss $L_{\mathrm{BPL}}$, the car penetration loss $L_{\mathrm{CPL}}$, the jumper and feeder losses, $L_{j}$ and $L_{f}$, the power control margin $P C_{m a r g}$, the log-normal fading margin $L N F_{m \text { arg }}$, and the antenna gain $G_{a}$, as given by 


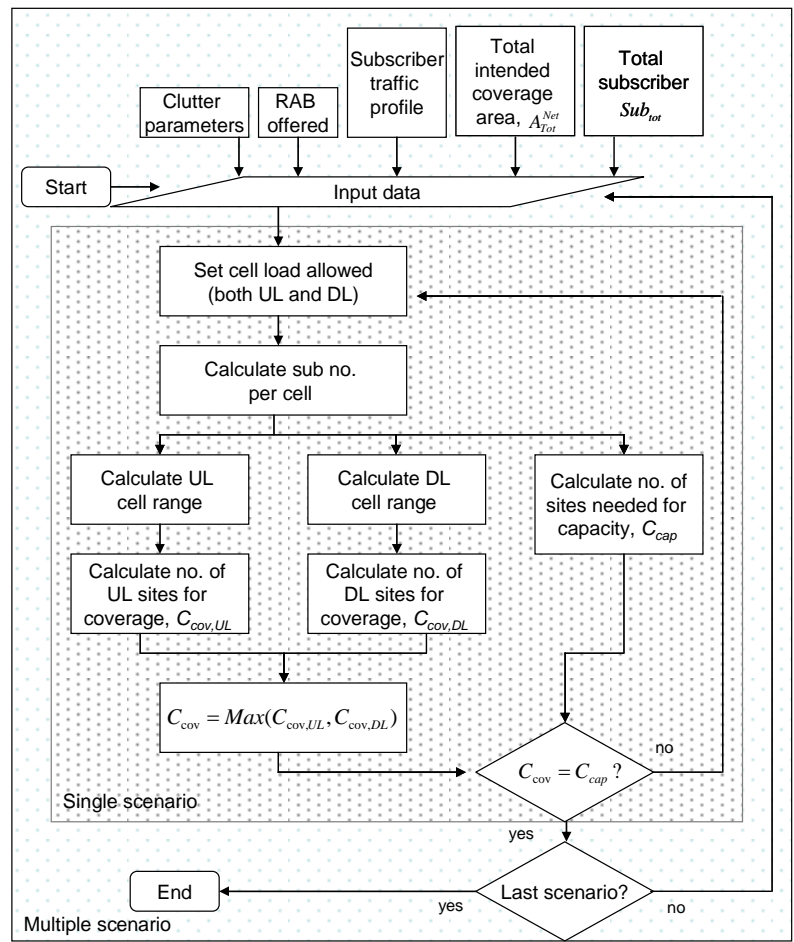

Figure 1. Existing dimensioning methodology.

$$
\begin{aligned}
G_{b}= & L_{\mathrm{BL}}+L_{\mathrm{BPL}}+L_{\mathrm{CPL}}+L_{j}+L_{f} \\
& +P C_{m \text { arg }}+L N F_{\text {marg }}-G_{a}
\end{aligned}
$$
by:

The base station receiver sensitivity $S_{\mathrm{UL}}$ [3] given

$$
\begin{aligned}
S_{\mathrm{UL}}= & N_{t, \mathrm{UL}}+N_{f, \mathrm{UL}} \\
& +10 \log R_{\mathrm{UL}, k}+\left(E_{b} / N_{0}\right)_{k, \mathrm{UL}}
\end{aligned}
$$

where $N_{t, \mathrm{UL}}$ is the thermal noise power spectral density, $N_{f, \text { UL }}$ is the base station noise figure, $R_{\mathrm{UL}, k}$ is the UL information bit rate of type $k$ service and $\left(E_{b} / N_{0}\right)_{k, \mathrm{UL}}$ is ratio of bit energy to noise spectral density for type $k$ service. The UL interference margin, $I_{\mathrm{UL}}$, is a function of the UL cell load [1], $\eta_{\mathrm{UL}}$ and is given by

$$
I_{\mathrm{UL}}=10 \log \left(\frac{1}{1-\eta_{\mathrm{UL}}}\right)
$$

The cell load is defined as the percentage of capacity utilized based on the theoretical maximum interference limit before interference reaches infinity. Capacity planning will be further elaborated in Section 2.2. An increase in cell load, $\eta_{\mathrm{UL}}$ will lead to a rise in noise level (i.e., increase in interference-plus-noise ratio due to own cell and adjacent cell) and results in higher $I_{\mathrm{UL}}$ as given in Equation (4), causing the radio coverage area to shrink as given in Equation (1). On the other hand, a decrease in cell load will result in a larger radio coverage area. As the cell load varies over time, the cell size increases or decreases from time to time, a phenomenon commonly known as cell breathing.

With $L_{\text {pathmax,UL }}$, the UL cell range $d_{\mathrm{UL}}$ can be obtained using an empirical model such as the OkumuraHata prediction model [1], given by

$$
d_{\mathrm{UL}}=10 \frac{L_{\text {path max }}-46.3-33.9 \log f_{c}+13.82 \log h_{b}+a\left(h_{m}\right)-C_{m}}{44.9-6.55 \log h_{b}}
$$

where

$$
a\left(h_{m}\right)=\left(1.1 \log f_{c}-0.7\right) h_{m}-\left(1.56 \log f_{c}-0.8\right)
$$

where $f_{c}$ is the carrier frequency in $\mathrm{kHz}, h_{b}$ and $h_{m}$

\begin{tabular}{|c|c|c|c|}
\hline Transmitter (mobile) & value & unit & remark \\
\hline MS output power, $P_{\mathrm{UE}}$ & 24 & $\mathrm{dBm}$ & $a$ \\
\hline Mobile antenna gain & 0 & $\mathrm{dBi}$ & $b$ \\
\hline Body loss, $L_{\mathrm{BL}}$ & 0 & $\mathrm{~dB}$ & C \\
\hline $\begin{array}{l}\text { Equivalent Isotropic Radiated } \\
\text { Power (EIRP) }\end{array}$ & 24 & $\mathrm{dBm}$ & $D=a+b$ \\
\hline Receiver (base station) & value & unit & remark \\
\hline Thermal noise density, $N_{t, \mathrm{UL}}$ & -174.0 & $\mathrm{dBm} / \mathrm{Hz}$ & $e$ \\
\hline Receiver Noise figure, $N_{f, \text { UL }}$ & 4.0 & $\mathrm{~dB}$ & $f$ \\
\hline $\mathrm{Eb} / \mathrm{No}_{k, \mathrm{UL}}$ & 2.7 & $\mathrm{~dB}$ & $g$ \\
\hline User rate, $R_{\text {info,UL }}$ & 67400 & bps & $h$ \\
\hline Required Signal Power, $B_{\text {sen }}$ & -118.99 & $\mathrm{dBm}$ & $\begin{array}{c}l=e+f+ \\
10 \log (h)+g\end{array}$ \\
\hline Interference margin, $I_{\mathrm{UL}}$ & 0.97 & $\mathrm{~dB}$ & $i$ \\
\hline $\mathrm{RX}$ antenna gain, $G_{a}$ & 18 & $\mathrm{dBi}$ & $j$ \\
\hline Feeder loss, $L_{j}$ & 3.2 & $\mathrm{~dB}$ & $k$ \\
\hline Coverage probability & $95 \%$ & & \\
\hline LNF margin, $L N F_{\mathrm{mrg}}$ & 8.98 & $\mathrm{~dB}$ & $t$ \\
\hline Power control margin, $P C_{\mathrm{mrg}}$ & 0.7 & $\mathrm{~dB}$ & $n$ \\
\hline Building penetration loss, $L_{\mathrm{BPL}}$ & 25.0 & $\mathrm{~dB}$ & $o$ \\
\hline Car penetration loss, $L_{\mathrm{car}}$ & 0.0 & $\mathrm{~dB}$ & $p$ \\
\hline Jumper and connector loss, $L_{j}$ & 1.2 & $\mathrm{~dB}$ & $q$ \\
\hline Total Gain and losses, $G_{b}$ & 20.1 & $\mathrm{~dB}$ & $\begin{array}{c}r=j-k-t-n- \\
\quad o-p-q-c\end{array}$ \\
\hline $\begin{array}{l}\text { Allowed Propagation Loss, } \\
L_{\text {pathmax,UL }}\end{array}$ & 121.94 & $\mathrm{~dB}$ & $s=d-l-r-i$ \\
\hline BS antenna height, $h_{b}$ & 35 & $\mathrm{~m}$ & \\
\hline MS antenna height, $h_{m}$ & 1.5 & $\mathrm{~m}$ & \\
\hline Cell range, $d_{\mathrm{UL}}$ & 0.4573 & $\mathrm{~km}$ & \\
\hline Coverage Area, $A_{\text {site }}$ & 0.41 & $\mathrm{Km} \cdot \mathrm{sq}$ & \\
\hline
\end{tabular}
denote the base station and mobile station antenna heights, respectively, and $C_{m}$ is the clutter attenuation factor. An illustration of UL radio link budget calculation is shown in Table 1.

Table 1. Example of UL link budget calculation for service [3] (3 km/h, dense urban area). 
The DL link budget can be calculated in the same way as the UL case where all subscripts UL in Equations (1)-(5) are replaced with subscript DL. In this case, $P_{\mathrm{DL}}$ is the base station output power, $S_{\mathrm{DL}}$ is the $\mathrm{UE}$ receiver sensitivity and $I_{\mathrm{DL}}$ is the DL interference margin. $G_{b}$ is a common parameter for both UL and DL and remains unchanged as given by Equation (2). With $L_{\text {pathmax,DL }}$, the cell range for DL $d_{\mathrm{DL}}$ can be obtained using an empirical model as given in Equation (5).

Assuming hexagonal cells, the coverage area for a 3sector site, $A_{\text {site } X}$ is given by:

$$
A_{\text {site }, X}=\frac{9}{8} \sqrt{3} d_{X}^{2}
$$

where $X \in\{\mathrm{UL}, \mathrm{DL}\}$. With the total intended coverage area, $A_{\text {Tot }}^{\text {Net }}$, the total number of sites needed for UL $\left(C_{\mathrm{cov}, \mathrm{UL}}\right)$ and DL $\left(C_{\mathrm{cov}, \mathrm{DL}}\right)$ can be calculated as

$$
C_{\mathrm{cov}, X}=\frac{A_{\mathrm{Tot}}^{\mathrm{Net}}}{A_{\text {site }, X}}
$$

The final site count for meeting the coverage requirement $C_{\text {cov }}$ is given by:

$$
C_{\mathrm{cov}}=\max \left(C_{\mathrm{cov}, \mathrm{DL}}, C_{\mathrm{cov}, \mathrm{UL}}\right)
$$

\subsection{Capacity Planning}

In a multi-service WCDMA system, different types of radio access bearers (RAB) are available, denoted by $k \in\left\{\right.$ voice, video, streaming, $P S_{\mathrm{UL} 1 / \mathrm{DL} 1}, P S_{\mathrm{UL} 2 / \mathrm{DL} 2}, \cdots$, $\left.P S_{\text {ULmax/DLmax }}\right\}$ where $P S_{\mathrm{ULn} / \mathrm{DL} n} \forall n=1,2,3 \cdots, n_{\max }$ are the packet-switched RAB of different $\mathrm{UL}\left(\mathrm{UL}_{n}\right)$ and $\mathrm{DL}\left(\mathrm{DL}_{n}\right)$ bit rates and $n_{\max }$ is the total number of packet-switched services. Each type of RAB has a different receiver signal-to-interference ratio (S/I) requirement for service $\mathrm{k}$ in $\mathrm{X}$ direction to maintain the link quality, and it is given by

$$
(S / I)_{k, X} \geq P \cdot g /\left(I_{b, X}+N\right)
$$

where $P$ is the transmit power assigned to the link, $g$ is the corresponding link gain, $I_{b, X}$ is the interference received and $N$ is the thermal noise. The UL will perform transmit power update in order to maintain the $(S / I)_{k, X}$ to achieve the required $\left(E_{b} / I_{o}\right)_{k, X}$ (energy-per-bit-tointerference-spectral-density ratio for service $k$ in $X$ direction) performance. The ratio between $\left(E_{b} / I_{o}\right)_{k, X}$ and $(S / I)_{k, X}$ can be expressed by

$$
\left(E_{b} / I_{o}\right)_{k, X}=G_{p, k, X} \cdot(S / I)_{k, X}
$$

where the subscript $X \in\{\mathrm{UL}, \mathrm{DL}\}$. If $N$ mobiles are connected to the same cell, the interference generated in the cell is given by

$$
I_{b, X}=\sum_{1}^{N} P_{X} \cdot g_{X}
$$

When the system is loaded, the interference level ex- perienced by each user's signal will increase as given in Equation (11). Since WCDMA systems have unity frequency reuse factor, the system is typically interference-limited. Thus, there is a theoretical limit to the number of simultaneous users that can be supported in a cell using a RAB. At this limit the interference level is perceived to be infinite, thus reducing the coverage to zero. The theoretical maximum number of UEs that can be supported in a cell for each type of service $(k)$ in $X$ direction, $N_{\text {user }, k, X}^{\max }$ is given by

$$
N_{\text {user }, k, X}^{\max }=\left(\frac{1}{1+F_{X}}\right)\left[\frac{G_{p, X}}{\left(E_{b} / I_{o}\right)_{k, X}}\right]
$$

where $F_{X}$ is the ratio between the interference from neighboring cells and own cell interference [3]. Thus when $N_{\text {user, } k, X}^{\max }$ users are admitted to the cell, the cell interference is perceived to be infinite and the cell is considered reaching maximum utilization or fully loaded. Thus, after the theoretical system capacity of a WCDMA cell is calculated, the cell load $\eta$ is defined as the percentage of capacity utilized based on the theoretical maximum interference limit.

In a cell serving different types of services, the total cell load $\eta_{X} \forall X \in\{\mathrm{UL}, \mathrm{DL}\}$, contributed by both circuit-switch services $\eta_{X, C S}$ and packet switched services $\eta_{X, P S}$ is given by

$$
\eta_{X}=\eta_{X, C S}+\eta_{X, P S}
$$

The cell load for circuit-switched services mainly comprises voice and video services

$$
\eta_{X, C S}=\frac{N_{\text {user,voice, } X}}{N_{\text {user,voice, } X}^{\max }}+\frac{N_{\text {user,video }, X}}{N_{\text {user,video }, X}^{\text {max }}}
$$

where $N_{\text {user,voice, } X}$ and $N_{\text {user,video, } X}$ are the number of simultaneous voice and video call users, respectively. The number of subscribers supported per cell, Sub $b_{X, \text { cell }}$ can be obtained as

$$
\eta_{X, C S}=\frac{N_{\text {user,voice }, X}}{N_{\text {user,voice }, X}^{\max }}+\frac{N_{\text {user,video }, X}}{N_{\text {user,video }, X}^{\text {max }}}
$$

where $S_{X \text {,voice }}$ and $S_{X \text {,video }}$ are the traffic offered in Erlang per subscriber for voice and video services, respectively.

The cell load contributed by a packet-switched service is given by

$$
\eta_{X, P S}=S u b_{X, \text { cell }} \times\left(\sum_{n=1}^{n_{\max }} \frac{S_{X, n} / R_{X, P S_{n}}}{N_{\mathrm{user}, P S_{\mathrm{UL}_{n} / \mathrm{DL}_{n}}, X}^{\max }} \times F_{\text {peak }}\right)
$$

where $S_{X, n}$ is the rate of packet data generated by each subscriber in kbps for packet-switched service types $n=1,2,3, \cdots, n_{\max }, R_{X, P S_{n}}$ is the system data rate for $n$ types of packet-switched service, $F_{\text {peak }}$ is a peak factor 
applied to each type of packet-switch service. The peak factor is applied as a margin to both UL and DL so that they are not overloaded when there is a surge in data arrival. Thus with each cell load level $\eta_{X}$, the number of subscribers supported $S u b_{X \text {, cell }}$ can be found by using Equations (13)-(16).

For capacity planning, based on the cell load and the number of subscribers supported per cell, the site count needed $C_{\text {cap }}$ to capture the total number of subscribers in the network, Sub $b_{\text {tot }}$ is given by

$$
C_{\text {cap }}=\frac{S u b_{\text {tot }}}{N_{c} \times N_{s} \times S u b_{\text {cell }}(\eta)}
$$

where $N_{c}$ and $N_{S}$ are the number of carrier frequencies and the number of sectors configured per site, respectively. Even with the same cell load level for both $\mathrm{UL}$ and DL $\left(\eta_{\mathrm{UL}}=\eta_{\mathrm{DL}}=\eta\right)$, the number of subscribers supported in the UL $\left(\operatorname{Sub}_{\mathrm{UL}, \text { cell }}(\eta)\right)$ and DL $\left(\operatorname{Sub}_{\mathrm{UL}, \text { cell }}(\eta)\right)$ might be different due to different values of $N_{\text {user, }, X}^{\max }$ in either direction as given in Equation (18). Thus, the number of subscribers supported per cell with a cell load of $\eta, \operatorname{Sub}_{\text {cell }}(\eta)$ is given by

$$
\operatorname{Su} b_{\text {cell }}(\eta)=\min \left[\operatorname{Su} b_{\mathrm{UL}, \text { cell }}(\eta), \operatorname{Su} b_{\mathrm{DL}, \mathrm{cell}}(\eta)\right]
$$

\subsection{Iterative Dimensioning Process}

To achieve balance in coverage and capacity planning, the site count needed to meet both the coverage and capacity requirements is calculated as shown in Figure 1. The dimensioning process starts by assuming maximum load and calculates the subscribers supported per cell. If the number of sites needed to meet the capacity requirement, $C_{\text {cap }}$, is less than the number of sites needed to meet the coverage requirement $C_{\text {cov }}$, calculations are repeated to gradually reduce the cell load, leading to fewer subscribers supported per cell according to Equation (15). This will lead to some noise reduction (i.e., a decrease in interference-plus-noise due to own cell and adjacent cells) which causes the radio coverage area to expand and thus fewer sites are needed to meet the coverage requirement. This process is repeated until further reduction of cell load allowed does not reduce the number of sites needed.

On the other hand, if the number of sites needed to meet the capacity requirement, $C_{\text {cap }}$, is more than the number of sites needed to meet the coverage requirement, $C_{\text {cov }}$, the cell load allowed needs to be increased gradually for both UL and DL as shown in Figure 1, leading to more subscribers supported per cell based on Equation (14) and fewer sites are needed to meet the capacity requirement. The dimensioning methods proposed in [1-3], [8] require coverage and capacity calculations (as shown in Figure 1) to be repeated iteratively by continuously adjusting the cell load allowed $\eta_{X} \forall X \in\{\mathrm{UL}, \mathrm{DL}\}$ until a trade-off between coverage and capacity requirements in terms of site count is obtained.

The process discussed above is applicable to singlescenario dimensioning. In reality, multiple scenarios are frequently encountered where the dimensioning process needs to be carried out for different types of clutter and cities each with different number of subscribers, area size, as well as subscriber behaviors. For example, urban subscribers tend to make longer voice calls than those in the rural area. The former lead to higher traffic in Erlang per subscriber, $S_{\text {voice }}$. Furthermore, operators may introduce different services in certain areas, e.g., mobile broadband is only introduced in certain residential areas. As a result, dimensioning needs to be carried out for each of these scenarios separately as each scenario will finally end up with different cell sizes and cell loads due to these diverse requirements. A separate dimensioning exercise for each of these scenarios is time consuming and requires a different team of engineers to work separately. To overcome this issue, a faster dimensioning method for the current cellular communication industry is desired.

\section{Proposed Dimensioning Methodology}

In this paper, we propose a dimensioning framework aimed to simplify multi-scenario dimensioning and reduce computational time without the need to execute the iterative process in the existing dimensioning approach. Figure 2 shows the flowchart of the proposed framework. The dimensioning framework splits the dimensioning process into two layers.

In the first layer, analysis of input data and quantification of cell coverage and capacity impact based on the subscriber and traffic profiles are carried out. Firstly, we reduce the complexity of the dimensioning process by avoiding the iterative process between coverage and capacity dimensioning. We first quantify the impact of traffic volume generated by each subscriber towards the cell load based on the input data. This is followed by normalization of subscriber traffic volume towards coverage area, and derivation of subscriber density supported by each level of cell load by separately considering coverage and capacity requirements. By quantifying the subscriber density of each level of cell load, the iterative process between coverage and capacity dimensioning for every scenario in multi-scenario network dimensioning exercises can be avoided. This method is further described in 3.1. Secondly; we reduce the repetition in a multi clutter dimensioning scenario by generalizing link calculation. This method is elaborated in Section 3.2.

In the second layer, the traffic input for each scenario is normalized and characterized. Based on the traffic characteristic, the minimum number of sites needed for a 


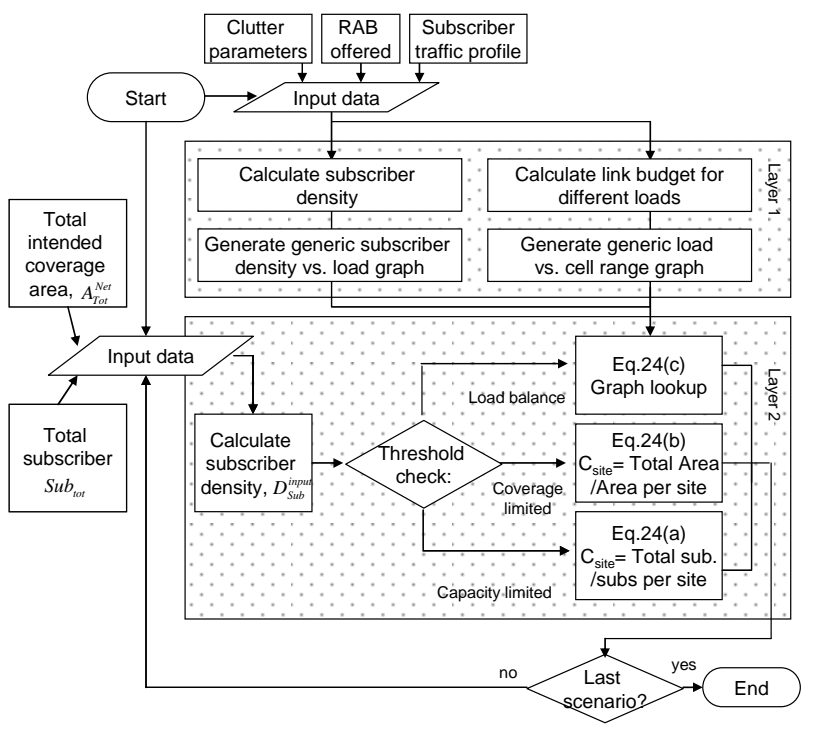

Figure 2. Proposed dimensioning methodology.

network can be obtained more efficiently from the result of network impact analysis prepared in layer 1 . This is further described in Section 3.3. In a multi-scenario dimensioning exercise, layer one is performed once and generic graphs are generated. Layer two is executed for every scenario and the dimensioning results can be obtained easily. The process is further described in Section 3.4. The proposed dimensioning methodology uses the same set of inputs used in the existing dimensioning method [1]. The inputs include the total intended coverage area, total number of subscriber, subscriber traffic profile and RAB offered, demographic input such as clutter parameters and coverage probability. Details of network impact analysis will be presented first, while traffic characterization and optimization for dimensioning of multiple scenarios will be discussed later in this section.

\subsection{Network Coverage and Capacity Impact Quantification}

As shown in Figure 3, the number of sites needed depends on the cell load. The existing dimensioning methodology iteratively adjusts the subscribers in each cell while evaluating the impact of cell load towards coverage and capacity until it finds an optimum solution where subscriber count per cell gives the minimum number of sites while fulfilling both coverage and capacity requirements. The impact of cell load towards coverage and capacity planning is given by Equation (4) and Equations (15) and (16), respectively.

Thus, to avoid using the iterative approach for evaluating the cell load, the impact of each subscriber with the respective traffic profile towards the cell load needs to be quantified first. For this purpose, we normalize the

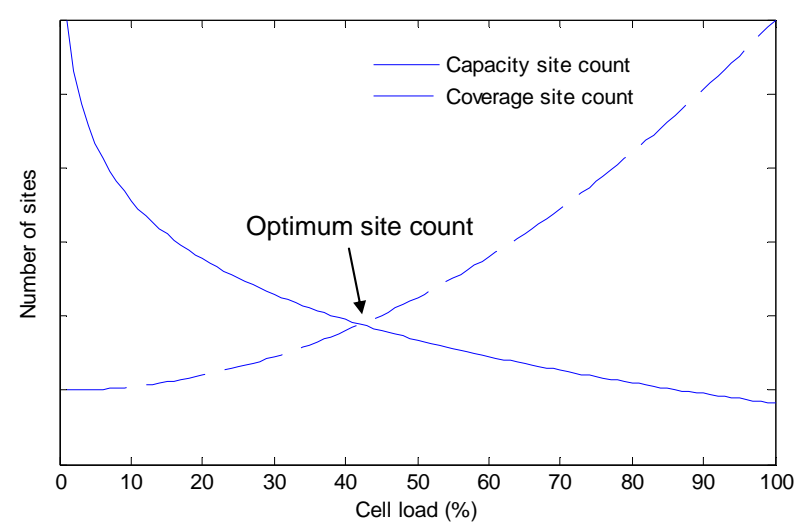

Figure 3. Number of sites required for coverage and capacity as a function of cell load.

subscriber number with the coverage area to obtain the subscriber density $D_{\text {Sub }}(\eta)$ of the area for each level of cell load, $\eta$. The number of subscribers supported per site with a specific load, $\operatorname{Sub}_{\text {site }}(\eta)$ is first calculated through capacity planning. This can be achieved by using Equations (12)-(16). On the other hand, the site coverage area resulting from a specific cell load, $A_{\text {site }}(\eta)$ is calculated in coverage planning, through Equations (1)-(6). Next, $\operatorname{Sub}_{\text {site }}(\eta)$ is normalized to $A_{\text {site }}(\eta)$ for each level of cell load $\eta$ to obtain the complete set of subscriber density $D_{\text {Sub }}(\eta)$, i.e.,

$$
D_{\text {Sub }}(\eta)=\frac{\operatorname{Sub}_{\text {site }}(\eta)}{A_{\text {site }}(\eta)}, \quad \text { where } \eta=[0,0.1, \cdots, 1]
$$

Since the subscriber density, $D_{\text {Sub }}(\eta)$ is a function of the site coverage area as given in Equation (19), different clutter types will result in different $D_{\text {Sub }}(\eta)$. By generalizing the clutter data, $D_{\text {Sub }}(\eta)$ is plotted on different loads for different types of clutter, and this is the only data needed for multi-scenario dimensioning. The result of $D_{\text {Sub }}(\eta)$ versus cell load is illustrated in Figure 4. In this example, each subscriber is assumed to consume 90 MB of data per month and two types of clutter are shown for both UL and DL where a $G_{b}$ margin (as given in Equation (2)) of $20.1 \mathrm{~dB}$ is used for dense urban clutter and a $G_{b}$ margin of $18.1 \mathrm{~dB}$ is used for urban clutter.

The total intended coverage area $A_{\mathrm{Tot}}^{\mathrm{Net}}$ and the total number of subscribers to be supported $S u b_{\text {tot }}$ serve as the input data for dimensioning. From the input data, the subscriber density of the intended coverage area, $D_{\text {Sub }}^{\text {input }}$ is given by

$$
D_{\text {Sub }}^{\text {input }}=\frac{S u b_{\text {Tot }}}{A_{\text {Tot }}^{\text {Net }}}
$$

With the input obtained from Equation (20), the final cell load for the scenario under study can be easily obtained by matching the subscriber density, as given by 


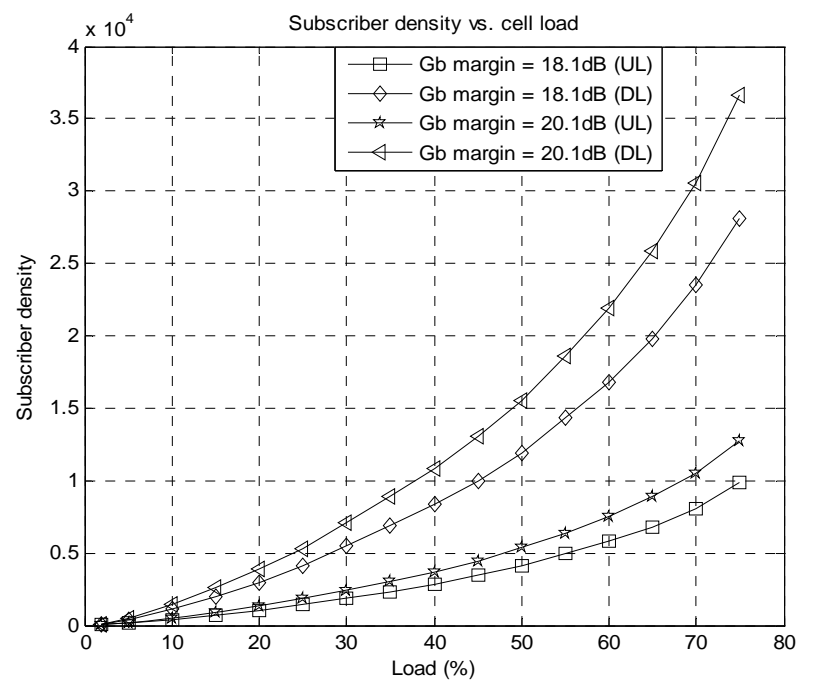

Figure 4. Subscriber density graph generated using the proposed methodology.

$D_{\text {Sub }}(\eta)=D_{\text {Sub }}^{\text {input }}$. With this proposed method, coverage planning and capacity planning can be separated by working individually on a common parameter, namely the cell load $\eta$. The number of sites needed, $C_{\text {site }}$ for an intended coverage area, $A_{\text {Tot }}^{\mathrm{Net}}$ is given by

$$
C_{\text {site }}=\frac{A_{\text {Tot }}^{\mathrm{Net}}}{A_{\text {site }}(\eta)}
$$

As coverage planning and capacity planning can be separated, the iterative approach of finding the trade-off between cell range and capacity requirement can be replaced by values lookup from the data generated separately from coverage and capacity planning using the normalized subscriber density, $D_{\text {Sub }}^{\text {input }}$.

\subsection{Improved Multi Clutter Dimensioning}

To further reduce computational time for multi-scenario dimensioning, link calculation is generalized to avoid repetitive calculations. In a typical dimensioning exercise, the demography of a coverage area can be classified into different clutters, e.g., dense urban, urban, and rural, each with different path loss parameters. The cell range is typically based on two important groups of parameters which are unique to each clutter type: 1 ) sum of all gains and losses between the base stations and mobile stations of different clutters, $G_{b}$ (each clutter will have its own $G_{b}$ value as given in Equation (2) and 2) $E_{b} / N_{o}$ value in both UL and DL as different types of service offered will have different coverage requirements. Thus, to further reduce computational time for multi-scenario dimensioning, coverage analysis is further extended to multiple clutters.

The impact of different clutters towards the coverage area, $A_{\text {site }}(\eta)$ is illustrated in Figure 5, where the cell range of different cell loads in different clutters is calculated from the link budget using Equations (1) to (7). Different clutter types are differentiated with their own $G_{b}$ values. With the different cell ranges $d_{X, k}$ calculated for service $k$ in both directions, $X \in\{\mathrm{UL}, \mathrm{DL}\}$, the cell range of the most stringent service (service with the least path loss) will be considered as the final cell range, i.e.,

$$
d=\min _{\substack{k \in \text { all services } \\ \mathrm{X} \in\{\mathrm{UL}, \mathrm{DL}\}}}\left(d_{X, k}\right)
$$

Thus, Figure 5 shows the final cell range, $d$ in each clutter is plotted for the most stringent service at different cell load levels, $\eta$ for both UL and DL. Similar to Section 3.1, two types of clutter are considered in this example where a $G_{b}$ margin of $20.1 \mathrm{~dB}$ is used for dense urban areas and a $G_{b}$ margin of $18.1 \mathrm{~dB}$ is used for urban clutter. With this generalization, only the cell range of the most stringent service (as given in Equation (22)) for different clutter will be used in multi-scenario dimensioning.

Once the sets of unique combination of subscriber density, cell load and cell range have been found, the cell range and cell load can be easily obtained when the subscriber density is specified using Figures $\mathbf{4}$ and $\mathbf{5}$ by following the steps below:

1) With the total number of subscribers, $S u b_{\text {Tot }}$ and total area, $A_{\text {Tot }}^{\text {Net }}$ as the inputs, the subscriber density $D_{\text {Sub }}^{\text {input }}$ is calculated.

2) With the subscriber density $D_{\text {Sub }}^{\text {input }}$, the cell load is obtained from the generic capacity graph (Figure 4).

3) With the cell load information, the cell range is evaluated from the generic coverage graph (Figure 5).

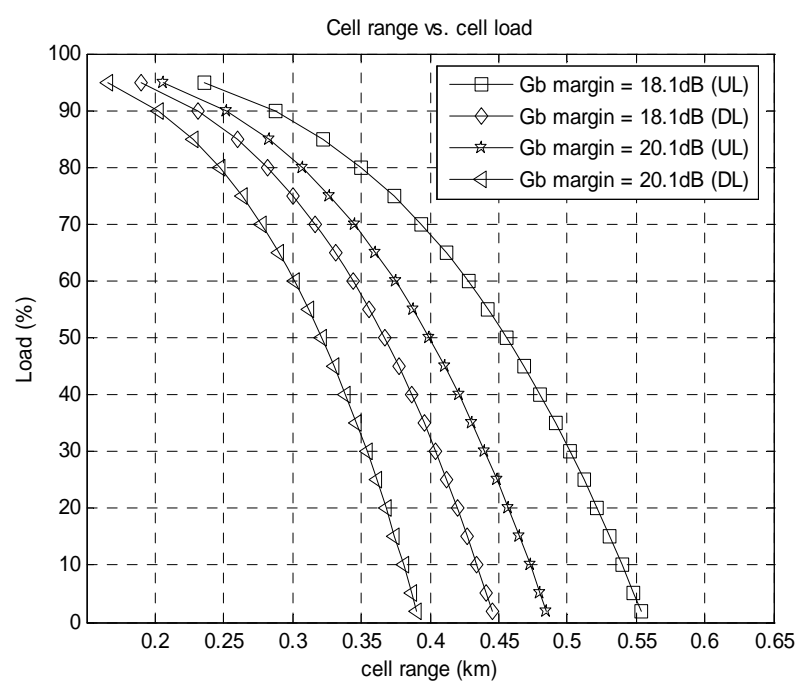

Figure 5. Cell load vs. cell range graph generated using the proposed methodology. 
It is observed that WCDMA systems are typically limited by uplink interference and result in higher UL load compared to DL in most of the cases.

The proposed method effectively allows coverage and capacity planning to be separated to reduce the number of iterations per scenario analysis. Furthermore, the proposed methodology greatly reduces computational time as the conventional dimensioning methodology needs to perform link calculation for every clutter type, every service for both UL and DL, and for each scenario in multi-scenario analysis.

\subsection{Network Characterization}

A greenfield macro site for WCDMA is typically unloaded and UL coverage limited as the power level of user equipment (UE) is limited to $125 \mathrm{~mW}$. In UL coverage-limited cases, the cell size is limited by the maximum path loss allowed in UL as given in Equation (1). Macro cell coverage is rarely limited in DL because the base station is typically equipped with a high power amplifier. On the other hand, low power sites such as micro and pico cells are typically DL coverage limited, where the base stations are typically equipped with a low-power amplifier and the cell size is limited by the maximum transmit power of the base station.

A loaded macro cell is typically limited by the available UL capacity of the cell; in particular, the UL interference capacity limit. The cell size will shrink when there are more users in the cell, as given by Equations (1) and (4). For UL capacity limited cases, the maximum cell capacity is limited by the maximum desired UL load, $\eta_{\mathrm{UL} \text {,max }}$ where the maximum number of subscribers supported per cell should not exceed $\operatorname{Su} b_{\mathrm{UL}, \text { cell }}\left(\eta_{\mathrm{UL}, \max }\right)$, as given in Equations (4) and (12)-(16). Similarly for the $\mathrm{DL}$, the subscribers supported per cell at the maximum desired DL load, $\eta_{\mathrm{DL} \text {,max }}$ should not exceed

$\operatorname{Sub}_{\mathrm{DL}, \mathrm{cell}}\left(\eta_{\mathrm{DL}, \max }\right)$.

In summary, the nature of the network can be characterized into different conditions as follows:

UL capacity limited:

$\left[d\left(\eta_{\mathrm{UL}, \max }\right)<d\left(\eta_{\mathrm{UL}}\right)\right] \cap\left[D_{\text {Sub }}^{\text {input }}>D_{\text {Sub }}\left(\eta_{\mathrm{UL}, \max }\right)\right]$

DL capacity limited:

$\left[d\left(\eta_{\mathrm{UL}}\right) \leq d\left(\eta_{\mathrm{UL}, \max }\right)\right] \cap\left[D_{\text {Sub }}^{\text {input }}>D_{\text {Sub }}\left(\eta_{\mathrm{UL}, \max }\right)\right]$

UL coverage limited:

$\left[d\left(\eta_{\mathrm{UL}, \min }\right)<d\left(\eta_{\mathrm{UL}}\right)\right] \cap\left[D_{\text {Sub }}^{\text {input }}>D_{\text {Sub }}\left(\eta_{\mathrm{UL}, \text { min }}\right)\right]$

DL coverage limited:

$$
\begin{gathered}
{\left[d\left(\eta_{\mathrm{UL}}\right) \leq d\left(\eta_{\mathrm{UL}, \min }\right)\right] \cap\left[D_{\text {Sub }}^{\text {input }}>D_{\text {Sub }}\left(\eta_{\mathrm{UL}, \min }\right)\right]} \\
\text { Load balanced condition: }
\end{gathered}
$$

Elsewhere.
In the second layer of the proposed dimensioning method, a threshold check is carried out to identify the network characteristic based on Equation (23). The number of sites can be found based on the value of the subscriber density to be supported as in Equation (24), i.e.,

$$
C_{\text {site }}= \begin{cases}\frac{S u b_{\text {tot }}}{N_{c} \times N_{s} \times S u b_{\text {cell }}\left(\eta_{X, \max }\right)}, & \text { capacity } \\ \frac{A_{\text {Tot }}^{\text {Net }}}{A_{\text {site }, X}\left(\eta_{X, \text { min }}\right)}, & \text { limited, } \\ \frac{A_{\text {Tot }}^{\text {Net }}}{A_{\text {site }, X}\left(\eta_{X}\right)} \text { where from } & \text { coverage } \\ \text { lookupgraph } & \text { limited, } \\ D_{\text {Sub }}\left(\eta_{X}\right)=\left(D_{\text {Sub }}^{\text {input }}\right), & \text { balanced } \\ & \text { case }\end{cases}
$$

The subscribers density specified by the requirement, $D_{\text {Sub }}^{\text {input }}$ which falls beyond the categories of UL capacity limited, DL capacity limited, UL coverage limited and DL coverage limited, will be classified as load balanced. A balanced cell load, $\eta$ can be looked-up from Figure 4. Where $D_{\text {Sub }}^{\text {input }}$ matches $D_{\text {Sub }}\left(\eta_{X}\right)$ and the cell range can be obtained from Figure 5. Once the cell range is determined, the number of sites required can be calculated using Equation (24).

\subsection{Optimization for Multiple Scenario Dimensioning}

With the generic coverage (Figure 5) and capacity (Figure 4) graphs generated, the information obtained in the initial scenario analysis can be reused in future scenarios analysis. Instead of performing repetitive top down calculation as in the existing network dimensioning practice, we propose to split the dimensioning process into two layers as shown in Figure 2. The information generated in the first layer is generic and can be reused to reduce computational time of multiple-scenario analysis. The second layer performs threshold checking on the input data to speed up computation, as the site count can be easily calculated if the input data fall within certain threshold before the graph lookup process takes place.

The first layer involves generating the generic coverage and capacity graphs as shown in Figure 2. In this layer, the generic graph as shown in Figures $\mathbf{4}$ and $\mathbf{5}$ is generated as mentioned earlier in Section 3.1. Calculation only needs to be done once and the calculated values can be reused for all other scenarios without repetitive calculations. In contrast to this proposed method, repetitive runs of single scenario dimensioning would require a new iteration of computation whenever there is a change in any network parameters regardless of whether it is related to coverage or capacity requirements. Besides the generic graph, the site coverage areas for minimum cell loads $A_{\text {site }, X}\left(\eta_{X, \min }\right)$ for both UL and DL, and the num- 
ber of subscribers per cell for maximum cell load in both directions, $\operatorname{Sub} b_{\text {cell }}\left(\eta_{X, \text { max }}\right)$ are calculated and passed to the second layer.

In the second layer, the nature of the network can be characterized into different conditions as given in Equation (23). In practice, the subscriber density information can be obtained easily either based on statistics or forecasted subscribers in a specific coverage area. With this proposed method, when the required subscriber density is provided, we can immediately determine the cell load density, the cell load and finally the cell range through the graph lookup process.

Apart from reducing computational time, the proposed method can give engineers better visualization on the effect of varying the subscriber density on the load density and how the cell range would be affected by using both the generic capacity and coverage graphs. With the generic coverage graph in place, whenever an RAB is introduced, or a change in channel model occurs, the number of sites needed can always be found and visualized easily using the proposed graph lookup method. With the generic capacity graph, a change in load per site as a new site is added, or a change in subscriber's voice or best effort traffic can easily be mapped into the new balanced cell range.

\section{Case Study and Analysis}

We evaluate the accuracy and efficiency of the proposed methodology through three case studies. In these case studies, dimensioning is carried out using both the existing and proposed methods and the outputs of dimensioning are compared to verify the accuracy of the proposed method. Furthermore, we also perform computational time analysis on both methods to verify the efficiency of the proposed dimensioning methodology. What-if analysis is also carried out to evaluate the flexibility of the proposed method.

\subsection{Case Study and Accuracy Analysis}

We consider three case studies of radio network planning for the Selangor state in Malaysia. These case studies are carried out in three different areas within the state, covering 34, 48, and $48.1 \mathrm{sq} \mathrm{km}$, respectively. Dense urban clutter is chosen for all three areas and the corresponding numbers of subscribers are $50 \mathrm{k}, 150 \mathrm{k}$ and $610 \mathrm{k}$, respectively. The radio design parameters include 95\% coverage probability, an antenna gain of $18 \mathrm{dBi}$, a building penetration loss of $25 \mathrm{~dB}$ and an antenna height of 35 $\mathrm{m}$. The voice and video traffic are assumed to be $25 \mathrm{mE}$ and $0.1 \mathrm{mE}$ per subscriber, respectively. Each subscriber is assumed to consume $90 \mathrm{MB}$ of data traffic per month. The minimum and maximum loads are assumed to be $10 \%$ and $60 \%$, respectively.
Dimensioning is carried out using both the existing method and proposed method. With the proposed method, the generic graph where subscriber density and cell range with various cell loads is first plotted, as shown in Figure 6. This generic graph is generated using the procedure described in Section 3 for the three case studies.

The results of dimensioning for these three case studies are shown in Table 2 and illustrated in Figure 6. Given Table 2, the subscriber density for the intended coverage area is first calculated. With $D_{\text {Sub }}^{\text {input }}$, the cell load $\eta$ can be obtained by matching $D_{\text {Sub }}^{\text {input }}$ with the supported subscriber density $D_{\text {Sub }}(\eta)$. Once $D_{\text {Sub }}(\eta)$ is obtained with its respective load, the cell range can be found. In case 1, both UL and DL loads are less than the minimum load threshold of $20 \%$. Thus, case 1 is characterized as a coverage limited scenario. The site count can be easily obtained through the network characterization method given in Section 3.3 simply by using Equation (24b). In Case 2, both UL and DL loads are within the minimum and maximum load thresholds. With the subscriber density $D_{\text {Sub }}(\eta)$, the cell range can be obtained from Figure 6. Therefore, Case 2 is characterized as load balanced and the site count can be obtained by using Equation (24c). In Case 3, both UL and DL loads exceed the maximum load threshold of $60 \%$. Thus, Case 3 is characterized as capacity limited and the site count can be obtained by using Equation (24a).

The accuracy of the results obtained with the proposed method has also been verified in Table 2, which compares the results obtained by the existing and the proposed methods. It is noted that the number of sites required to fulfil the coverage and capacity requirements using the proposed method are shown to be identical to that found using the existing method.

Thus the key strength of the proposed method is that provides quick and yet accurate dimensioning results for all types of scenarios: coverage limited, capacity limited, as well as load balanced networks.

\subsection{What-If Analysis on Individual Cell}

As shown in Figure 6, when the subscriber density is doubled from case 1 to case 2, the cell behavior changes from DL coverage-limited to DL cell balanced. By doubling the number of subscribers, the cell range shrinks $4 \%$ from $380 \mathrm{~m}$ to $365 \mathrm{~m}$. This translates to $8 \%$ more sites needed to cover the same area due to the cell breathing effect.

On the hand, when the subscriber density is increased four times from case 1 to case 3, the cell behavior changes from DL coverage limited to DL capacity limited. By quadrupling the number of subscribers, the cell will load up by $60 \%$ and the cell range will shrink $20 \%$ from 380 $\mathrm{m}$ to $300 \mathrm{~m}$. This translates to $60 \%$ more sites needed 


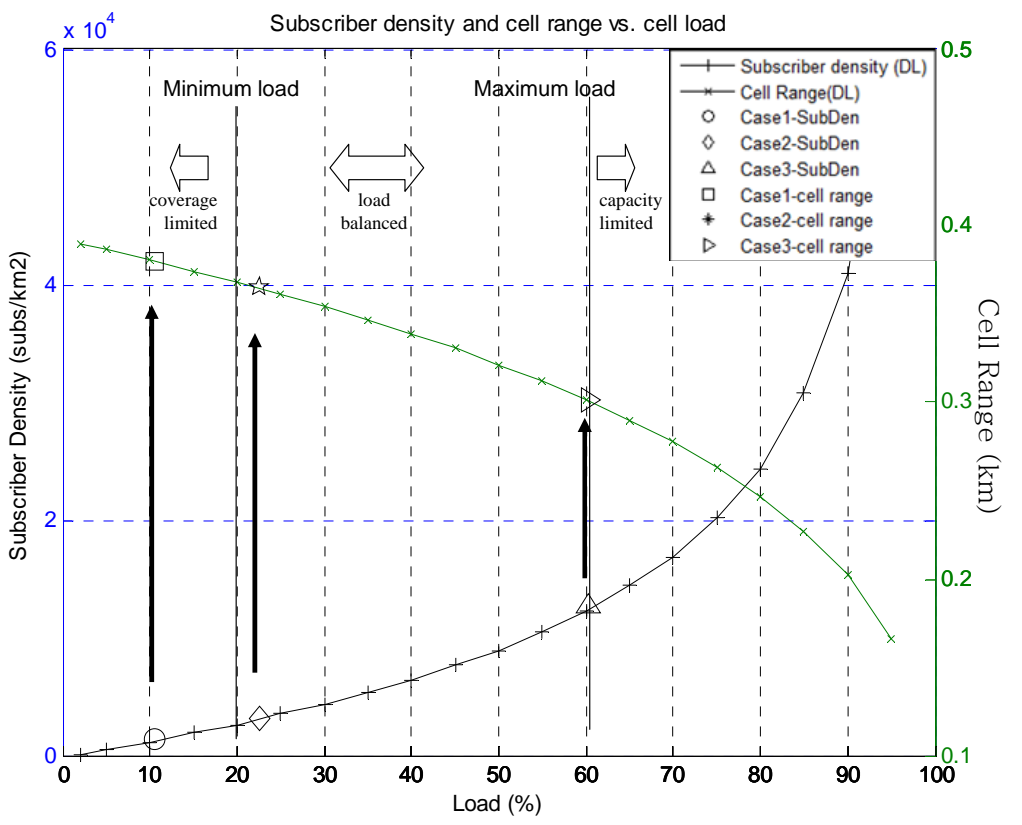

Figure 6. Illustration of network characterization and graph lookup to obtain the cell range.

Table 2. Dimensioning output analysis and comparison between the existing and proposed dimensioning methods.

\begin{tabular}{|c|c|c|c|}
\hline & Case 1 & Case 2 & Case 3 \\
\hline Subscriber density & $1470 / \mathrm{km}$ square & 3125/km square & $12708 / \mathrm{km}$ square \\
\hline Cell load & $\mathrm{UL}=15.7, \mathrm{DL}=10.5$ & $\mathrm{UL}=30.5, \mathrm{DL}=22.5$ & $\mathrm{UL}=71.7, \mathrm{DL}=60.3$ \\
\hline cell range (km) & $\mathrm{UL}=0.457, \mathrm{DL}=0.380$ & $\mathrm{UL}=0.434, \mathrm{DL}=0.365$ & $\mathrm{UL}=0.339, \mathrm{DL}=0.301$ \\
\hline Analysis & $\begin{array}{l}\text { Both UL and DL loads are less } \\
\text { than the minimum load threshold }\end{array}$ & $\begin{array}{l}\text { Both UL and DL loads are within the } \\
\text { minimum and maximum load thresholds }\end{array}$ & $\begin{array}{l}\text { Both UL and DL load exceed the } \\
\text { maximum load threshold. }\end{array}$ \\
\hline Classification & UL and DL coverage limited & Coverage and capacity balanced & DL capacity limited \\
\hline \multirow{2}{*}{$\begin{array}{l}\text { Number of sites required } \\
\text { based on the proposed } \\
\text { method }\end{array}$} & Total area & \multirow{2}{*}{$\begin{array}{l}\text { From the lookup graph, the cell range is } \\
\text { obtained and the site area is calculated. } \\
\text { The total site count is given by } 34 / 0.259 \\
=185 \text { sites }\end{array}$} & Total sub no \\
\hline & $\begin{array}{r}\text { Area per site } \\
=121 \text { sites }\end{array}$ & & $\begin{array}{l}\text { sub no per site at max load } \\
=610,000 / 2243=272 \text { sites }\end{array}$ \\
\hline $\begin{array}{l}\text { Number of sites based on } \\
\text { the existing method }\end{array}$ & 121 sites & 185 sites & 272 sites \\
\hline
\end{tabular}

to cover the same area purely driven by capacity. However, a $60 \%$ cell load is still not sufficient to handle the traffic due to high subscriber density, and more sites are needed to handle this excessive capacity. By putting up more macro sites to accommodate this capacity increase might not be cost effective, other solutions such as pico cells can be considered. The proposed methodology enables quick what-if analyses and gives engineers an insight into network's behavior, which could be useful to make informed decisions in handling high traffic.

\subsection{Computational Time Analysis}

In this section, we compare the computational time of both the proposed and existing dimensioning methods. Both methods are implemented in MATLAB 7.0 on a Pentium III $1.13 \mathrm{GHz}$ computer with a $256 \mathrm{M}$ memory running on Windows XP operating system.
The three case studies in Section 4.1 are extended from one to four clutter types, with 100 cities and five phases covering various parts of Malaysia. Four different types of clutter ( $n_{\text {Clutter }}=4$ ), namely dense urban, urban, suburban and rural areas are considered in each of the 100 cites for all five phases. Thus, 2000 scenarios are considered in this exercise to evaluate the computational time of both methods. Figure 7 shows that the proposed two-layer approach requires much shorter computing times as compared to the existing method. In fact, a substantial reduction in computational time can be achieved by the proposed method, particularly when $n_{\text {Cluter }} \times n_{\text {City }}$ $\times n_{\text {Phase }}$ is large. It is also observed that the existing method requires computational time that increases linearly with $n_{\text {Clutter }} \times n_{\text {City }} \times n_{\text {Phase }}$, while the proposed method only needs a short and almost constant computational time. Thus, this shows that the proposed dimen- 


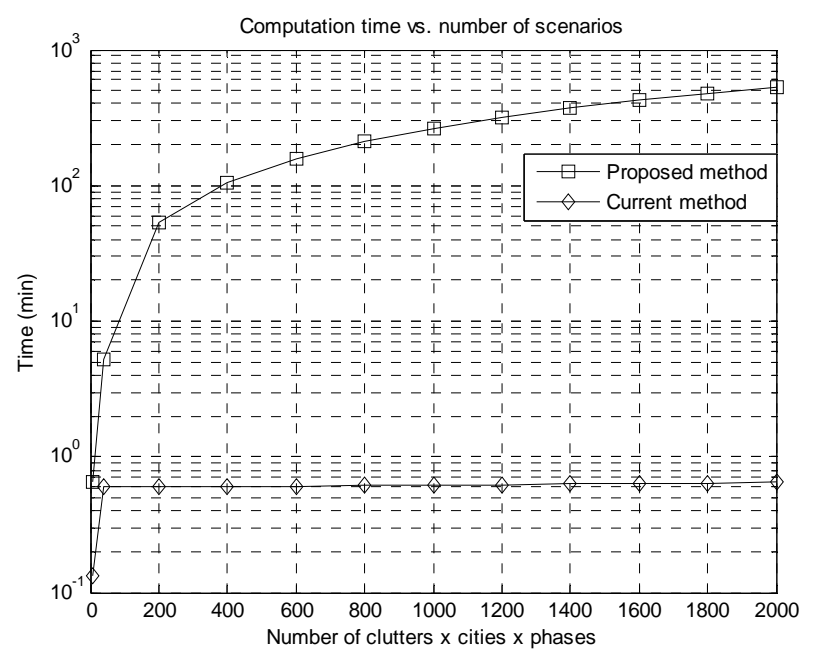

Figure 7. Computational time comparison between the existing and proposed methods.

sioning method is more efficient for multiple-scenario radio network dimensioning.

\section{Conclusion}

In this paper, a two-layer dimensioning method which can reduce the computational time of WCDMA radio network planning has been proposed. The first layer involves generation of generic graphs, while the second layer consists of threshold filtering and graphs lookup. Simulation and case study results show that the planning and what-if analysis time of the proposed dimensioning approach are independent of the number of scenarios considered while the time taken by the existing method increases linearly with the number of scenarios considered. In addition, the proposed approach can give better visualization for engineers in handling design parameters and perform what-if analysis when user behaviour changes. This method can be effectively and efficiently used by radio network operators or planners to evaluate vari- ous network dimensioning proposals of different design considerations.

\section{REFERENCES}

[1] J. Laiho, A. Wacker and T. Novosad, "Radio Network Planning and Optimisation for UMTS,” Wiley, Hoboken, 2006.

[2] B. Olin, H. Nyberg and M. Lundevall, "A Novel Approach to WCDMA Radio Network Dimensioning," Proceedings of Vehicular Technology Conference, Vol. 5, 26-29 September 2004, pp. 3443-3447.

[3] S. S. Igor, "WCDMA Radio Access Network Dimensioning for Multiple Services," Proceedings of 12th Telecommunications Forum, Belgrade, November 2004.

[4] A. Masmoudi and S. Tabbane, "Air Interface Dimensioning Techniques for Coverage and Capacity-Limited HSPA Based Networks," 17th International Conference on Telecommunications, Doha, 4-7 April 2010, pp. 196-203.

[5] A. A. Khalek, L. Al-Kanj, Z. Dawy and G. Turkiyyah, "Optimization Models and Algorithms for Joint Uplink/ Downlink UMTS Radio Network Planning With SIRBased Power Control," IEEE Transaction on vehicular technology, Vol. 60, No. 4, 2011, pp. 1612-1625.

[6] B. S. Northcote and N. A. Tompson, "Dimensioning Telstra's WCDMA (3G) Network," 15th IEEE International Workshop on Computer Aided Modeling, Analysis and Design of Communication Links and Networks, Miami, 3-4 December 2010, pp. 81-85.

[7] R. Pierre, F. Hoefel and G. Braz, "Throughput and Coverage Analysis of WCDMA and HSDPA Networks," Fifth International Conference on Digital Telecommunications, Athens, 13-19 June 2010, pp. 70-76.

[8] H. Holma and A. Toskala, "HSDPA/HSUPA for UMTS," John Wiley \& Sons Ltd., Hoboken, 2006. doi:10.1002/0470032634

[9] S. Dharmaraja, V. Jindal and U. Varshney, "Reliability and Survivability Analysis for UMTS Networks: An Analytical Approach,” IEEE Transaction on Network and Service Management, Vol. 5, No. 3, 2008, pp. 132-142. 